\title{
HETEROGENEOUS NUCLEATION AT A SINGLE DEFECT AND SUBSEQUENT GROWTH OF MARTEN- SITIC PHASE TRANSITIONS
}

\author{
R.J. GOODING and G.S. BALES \\ Dept of Physics, Queen's University, Kingston, Ontario Canada K7L 3N6
}

\begin{abstract}
A description of heterogeneous nucleation at a single defect, followed by the evolution of the nucleus as it grows in the parent phase background, is given. The theory is based on a nonlinear and nonlocal elastic free energy expansion, and the main assumption in our treatment is that of the interface separating parent and martensitic phases being completely coherent. The nucleating event is simply the response of the parent phase to the stress field of a defect that couples to the order parameter strain describing the transition. At the first-order transition temperature, around a weak defect, a small strain is produced. Then, after supercooling the system through the bulk transition temperature, a martensitic nucleus may appear, and we follow its growth as described by the deterministic dynamics of the elastic field. We show that the growth is going to lead to a twinned product phase due to dynamical energy reasons, viz. minimizing the kinetic energy, in contrast to the static energetics arguments of nonlinear elastic theories. Thus, this evolution of a martensitic embryo provides a simple and natural explanation of the phenomenon of autocatalytic twin formation.
\end{abstract}

\section{$\S$ I. Introduction}

A martensitic phase transition can be thought of as a first-order structural change of a solid that is both diffusionless, and involves some component(s) of the strain tensor as the primary order parameter /1/. Due to the diffusionless character of such transitions, one can envision that the lattice does not suffer any topological changes, namely that "no bonds are broken" as the transition is traversed, and thus (apart from hysteresis considerations) the transition is effectively reversible. Consequently, one must consider all inhomogeneous structures present in any intermediate temperature regime to have completely coherent interfaces. In this report we shall adopt this coherent structures' approximation, and shall thus present a complete description of the energetics associated with a single nucleation event at a defect site, thus leading to a completely coherent martensitic nucleus, as well as the subsequent growth of the nucleus to a final product phase consisting of twinned martensite. This will provide a natural explanation of the autocatalytic formation of twins based entirely on dynamical considerations.

It is prudent to qualify the limitations of the work presented here, since only a small part of the martensitic nucleation and growth problem is now purported to be understood. Firstly, at the experimental level it has not been established whether or not the martensitic nucleus parent phase background matrix interface is necessarily coherent. This is due, in part, to the highly localized nature of the nucleus, and thus the direct observation of a single nucleation event has seemed all but impossible. A possible breakthrough in this area is the TEM studies of Tanner et al. /2/ examining the development of the 7M phase of NiAl alloys in the region of a cracktip. The cracktip represents a large and highly localized stress field around which a localized martensitic embryo develops. Using high-resolution electron microscopy the interface 
separating the $7 \mathrm{M}$ embryo and the $\mathrm{CsCl}$ parent phase may be examined, and the interface is found to be at least semi-coherent $/ 2 /$. Unfortunately, studies at different temperatures that would follow the development of the embryo have yet to be done. This is only one particular martensitic transition, one that is moderately first order /1/. Thus it seems likely the model employed here, based on completely coherent interfaces, does describe the transitions that are at least weakly first order $/ 1$ ( (for which interfacial free energies are the smallest), but certainly not all. The second limitation of our report is that even though we shall choose the 3D tetragonalorthorhombic transition as the simplest prototypical symmetry-breaking, first-order martensitic transition that can be studied theoretically, here we shall only describe one-dimensional nucleation and growth. Thus, we shall not cloud our discussion with the complications arising from the necessity of satisfying the compatability relations $/ 3 /$, nor the morphological constraints imposed by the accommodation problem $/ 4 /$, even though very similar work may be carried out for higher dimensions. Lastly, we shall use a nonlinear, nonlocal elastic free-energy density, and thus the effect of temperature is included in the mean-field sense that it enters into any Landau-type theory; fluctuations are strictly ignored.

\section{§II. Heterogeneous Nonclassical Nucleation at a Single Defect}

Our presentation of the effect of a defect on the local free energies is largely based on the formalism presented elsewhere $/ 5 /$, and thus only the salient points shall be discussed here. However, even with this identical formalism we shall propose a very different explanation of the nucleating event.

As the simplest model system for which we may describe the nucleation and growth processes, we consider the tetragonal-to-orthorhombic transition. Of course, this is equivalent to describing the square-to-rectangular transition in two dimensions with the deviatoric strain, as formulated by Jacobs $/ 6 /$, and Barsch and Krumhansl $/ 7 /$. This strain is given by

$$
e_{2} \equiv \frac{1}{2}\left(e_{x x}-e_{y y}\right)
$$

for which the Landau-type elastic free-energy density of a pure defect-free system may be taken to be

$$
\begin{aligned}
F & =\int \tilde{F} d V \\
\tilde{F}^{0} & =\frac{A}{2} e_{2}^{2}-\frac{B}{4} e_{2}^{4}+\frac{C}{6} e_{2}^{6}+\frac{G}{2}\left(\vec{\nabla} e_{2}\right)^{2}
\end{aligned}
$$

where $B, C$, and $G$ are all strictly positive. The Landau parameter $A$ appearing in Eq. (2) is equivalent to the $\left(c_{11}-c_{12}\right)$ elastic constant which we assume to be temperature dependent in the usual fashion:

$$
A=|\alpha|\left(T-T_{c}\right) \text {. }
$$

Then, the local potential has a temperature dependence such that four different shapes of potentials are found $/ 6 /$. Above the metastability temperature $T_{m s}$, the only state with local stability is the unstrained $e_{2}=0$ state. Between the first-order transition temperature $T_{1}$ and $T_{m s}$, the unstrained state is still the unique stable state, but two metastable states appear at nonzero strains $\pm e_{M}(T)$. These two states represent the doubly degenerate rectangular phase. Then, between $T_{1}$ and $T_{c}$, the rectangular phases are stable, while the unstrained square phase is metastable. Then, at the instability temperature $T_{c}$ the square phase loses metastability and only the two rectangular states are stable. The strain of the rectangular phases is given by

$$
e_{M}^{2}(T)=\frac{B}{2 C}\left[1+\sqrt{1-\frac{4 C|\alpha|}{B^{2}}\left(T-T_{c}\right)}\right] .
$$

As mentioned above, we shall only allow for spatial variations of the strain along the $(1,1)$ (or equivalently the $(1,-1)$ ) direction, for which the compatability relations $/ 3 /$ are satisfied, and 
thus only one-dimensional structures (e.g., twin bands) are studied. We shall refer to the $(1,1)$ direction as $\eta$.

In order to incorporate a single defect we must append the free-energy density with the external stress tensor arising from the defect. Thus we take

$$
\tilde{F}=\tilde{F}^{0}-\sigma e_{2}
$$

where $\sigma$ is the component of the stress tensor (possibly spatially varying) that couples to the primary order parameter. A variety of forms of this stress field have been examined /5,8/, and those used in these papers were based on the total stress that responds to the defect, as found for example in linear elastic theory /9/. Here we shall enforce that the stress that is encorporated into Eq. (5) is correctly that representing the body forces acting on the parent phase background, and thus is restricted to act only from the surface of the defect. To be specific, and we emphasize, this is the external stress. As an unimportant simplification /10/, but one that allows for simple analytical progress to be made, we shall take the defect to be a point source, and thus we use

$$
\sigma(\eta)=\sigma_{0} \delta(\eta)
$$

for which the field equation describing static zero force strain configurations is given by

$$
G \frac{\partial^{2} e_{2}}{\partial \eta^{2}}=A e_{2}-B e_{2}^{3}+C e_{2}^{5}-\sigma_{0} \delta(\eta)
$$

Given the temperature dependence prescribed in Eq. (3), one need only examine the solutions of Eq. (7) to follow the response of the system to the defect at any temperature. It will be expedient for us to first examine the allowed solutions at the first-order transition temperature $T_{1}$; for very weak defects the appearance of a heterogeneous nucleus is trivially accounted for. At $T_{1}$, the minimum energy solution of Eq. (7) is given by

$$
e(\eta)=\frac{e_{M}\left(T_{1}\right)}{\sqrt{1+D \exp (|\eta| / L)}}
$$

where $e_{M}\left(T_{1}\right)$ follows from $E q$. (4), and $L^{2}=G / 4 A$. The constant $D$ is determined by choosing the largest value of $D$ that is a solution to

$$
\frac{D}{(1+D)^{3 / 2}}=\frac{L \sigma_{0}}{e_{M}\left(T_{1}\right) G} .
$$

In particular, all solutions must have $D>2$. For very small stresses produced by the defect, it then follows that the minimum energy strain field is given by

$$
e(\eta)=\frac{e_{M}\left(T_{1}\right)}{\sqrt{1+\left(\frac{e_{M}\left(T_{1}\right) G}{L \sigma_{0}}\right)^{2} \exp (|\eta| / L)}}
$$

Thus $e(0) \ll e_{M}\left(T_{1}\right)$ is the strain that develops at the impurity; it clearly increases as the strength of the impurity increase.

We now introduce the spinodal strain, which is defined by

$$
\epsilon_{s s}(T)=\frac{3 B-\sqrt{9 B^{2}-20 A C}}{10 C} .
$$

This strain represents the limit of linear stability; for strains which just exceed this value, sound waves excitations are unstable and the system must proceed to grow into some new phase. For 
martensitic nucleation to occur, somewhere in the crystal the strain must exceed this value. Now note that the strain that develops in the system at a weak defect, viz. $e(0)$ in Eq. (8), will not exceed the spinodal strain for sufficiently small $\sigma_{0}$. If the system is cooled to below $T_{1}$ the strain field solving Eq. (7) will be different than in Eq. (8), and one finds that a larger strain at the defect develops as the temperature is lowered /10/. Thus, only with undercooling the system beyond $T_{1}$ can the strain approach the spinodal strain for a weak defect $/ 10 /$, and thus eventually that of the martensitic phase. An alternative mechanism by which $e(0)$ can approach the spinodal strain is by thermal activation. This, however, is seen to be different than purely kinetic nucleation theories, since here the energy barrier to nucleation will be dependent on where in the crystal one is looking, and will be much smaller around the defect due to the increased strain found at the defect, as seen from Eq. (8). It is in either of these ways that a heterogeneous route to martensitic nucleation could be accomplished by a weak defect.

\section{§III. Interfacial Growth of Martensite: Autocatalytic Twin Formation}

We now suppose that a system with defects that couple to the strain field is cooled below $T_{1}$ (but above $T_{c}$ ), and that the most potent defect, viz. the defect with the largest $\sigma_{0}$, has locally developed a strain profile that represents the martensitic nucleus (again, assuming completely coherent interfaces). We thus address the following question, how does the nucleus grow to form the martensitic product phase? In order to address this question we adopt the simplest approach possible - we simply integrate the equations of motion describing the elastic field.

Utilizing the nonlinear, nonlocal elastic energy density given in Eq. (2), the kinetic energy density given by

$$
T=\frac{1}{2} \rho\left(\frac{\partial u}{\partial t}\right)^{2}
$$

where $u$ is the displacement field, and the dissipation function /11/ that includes the viscosity of the sound waves, viz.

$$
R=\frac{1}{2} \gamma\left(\frac{\partial e}{\partial t}\right)^{2}
$$

the equation of motion governing the evolution of the strain is given by

$$
\rho \frac{\partial^{2} e}{\partial t^{2}}=\frac{\partial^{2}}{\partial \eta^{2}}\left[A e-B e^{3}+C e^{5}-G \frac{\partial^{2} e}{\partial \eta^{2}}+\gamma \frac{\partial e}{\partial t}\right] .
$$

As the initial state of the system, we choose a pulse representing the martensitic nucleus, for example

$$
e(\eta, t=0)=e_{M}\left(T_{1}\right) \exp \left(-\eta^{2}\right) .
$$

(Note that the inclusion of the impurity stress introduced in Eq. (6) is irrelevant once the nucleus has appeared.) Since the system is symmetric about $\eta=0$, we only follow temporal development of the $\eta>0$ portion of the crystal.

The results obtained from this simple procedure are fascinating - for small sound-wave viscosity (such as occurs in nearly all physical systems), and sufficiently large undercooling, the evolution of the strain is shown in the Figure. The initial pulse autocatalytically twins, and then continues to twin in a completely regular fashion, and the system evolves into a fully twinned product phase - that there is equal length of the 2 twinned regions, in contrast to the crystallographic morphological theory $/ 12 /$, is a byproduct of us considering one-dimensional growth.

The reason for the autocatalytic twinning behaviour is easily understood if the displacement field is found from an integration of the strain field - if a single interface simply propagated throughout the system, the kinetic energy would be infinite. To be specific, suppose the pulse of Eq. (14) simply developed such that the strain at all times was always positive. For example, a propagating interface $/ 13 /$ of the type $(\eta>0)$

$$
e(\eta, t)=\frac{e_{M}\left(T_{1}\right)}{\sqrt{1+\exp (\eta-v t)}}
$$


could be imagined. Then, the difference between the displacement fields at the boundary, given by

$$
u(\infty, t)-u(-\infty, t)=\int_{-\infty}^{\infty} e(\eta, t) d \eta
$$

would always be an increasing function of time - this corresponds to the entire crystal being in motion, and thus an infinite kinetic energy. In contrast to this, for the strain evolving as shown in the Figure, the right-hand side of Eq. (15) is always zero, viz. the boundaries remain fixed. So, by having the interface perpetually twin, the kinetic energy of the system is always localized, and thus the minimum energy path is chosen by the solutions of Eq. (13). We caution that this autocatalytic twin production depends on the degree of undercooling and the damping in the system, and will be discussed in full detail elsewhere $/ 13 /$. Previous theories of autocatalytic twin formation /14/ have focussed on the nonlinear elastic energy, which of course is very important to the stabilization of the martensitic phases, while our explanation of this behaviour is completely dynamical in nature.

\section{$\S$ IV. Summary:}

We have begun our treatment of martensitic nucleation in weakly first-order systems by making the assumption that the interfaces that exist in the system in any inhomogeneous intermediate state are completely coherent, something of present concern to experimentalists $/ 2 /$. Then, using the square-to-rectangular model as an example, we have shown how the system responds to a weak point defect; complete details of the strain system's response away from $T_{1}$ to the impurity will be published elsewhere $/ 10 /$. By undercooling the system through $T_{1}$ a martensitic nucleus must appear, and we have followed the evolution of the nucleus using completely deterministic dynamics including the viscosity of the sound waves. We find that a twinned product phase results from the autocatalytic twinning that occurs at the interface, a consequence of localizing the kinetic energy in the system: further details of the system's dynamics will also be published elsewhere /13/.

We thank Lee Tanner for important comments on the experimental studies of a defectinduced nucleus. This work has been supported by the Natural Sciences and Engineering Council of Canada, and the Advisory Research Council of Queen's University.

\section{References:}

1. L.E. Tanner, and M. Wuttig, Mat. Sci. Eng. A127 (1990) 137.

2. L.E. Tanner, D. Schryvers, and S.M. Shapiro, Mat. Sci. Eng. A127 (1990) 205; L.E. Tanner (private communication).

3. A.E. Love, The Mathematical Theory of Elasticity (Dover, New York, 1944).

4. A.L. Roitburd, Solid State Physics 33 (1978) 317.

5. W. Cao, J.A. Krumhansl, and R.J. Gooding, Phys. Rev B41 (1990) 11319.

6. A.E. Jacobs, Phys. Rev. B31 (1985) 5984.

7. G.R. Barsch, and J.A. Krumhansl, Met. Trans. A19 (1988) 761.

8. W. Cao, and J.A. Krumhansl, Phys. Rev. B42 (1990) 1324.

9. J.D. Eshelby, Proc. Roy. Soc. London, Ser. A 241 (1957) 376; ibid, 252 (1959) 561; P.H. Dererichs, and J. Pollman, Z. Phys 255 (1972) 315.

10. R.J. Gooding and G.S Bales (submitted to Phys. Rev. B).

11. L.D. Landau, and I.M. Lifshitz, Theory of Elasticity (Pergamon, New York, 1982).

12. A.G. Khachaturyan, Theory of Structural Phase Trabsformations in Solids (Wiley, New York, 1983).

13. G.S Bales, and R.J. Gooding (submitted to Phys. Rev. Lett.).

14. L. Delaey, G. Guenin, Y. Murakami, P. Gobin, G. van Tendeloo, and J. van Landuyt, Phys. Stat. Solidi, A87 (1985) 457. 

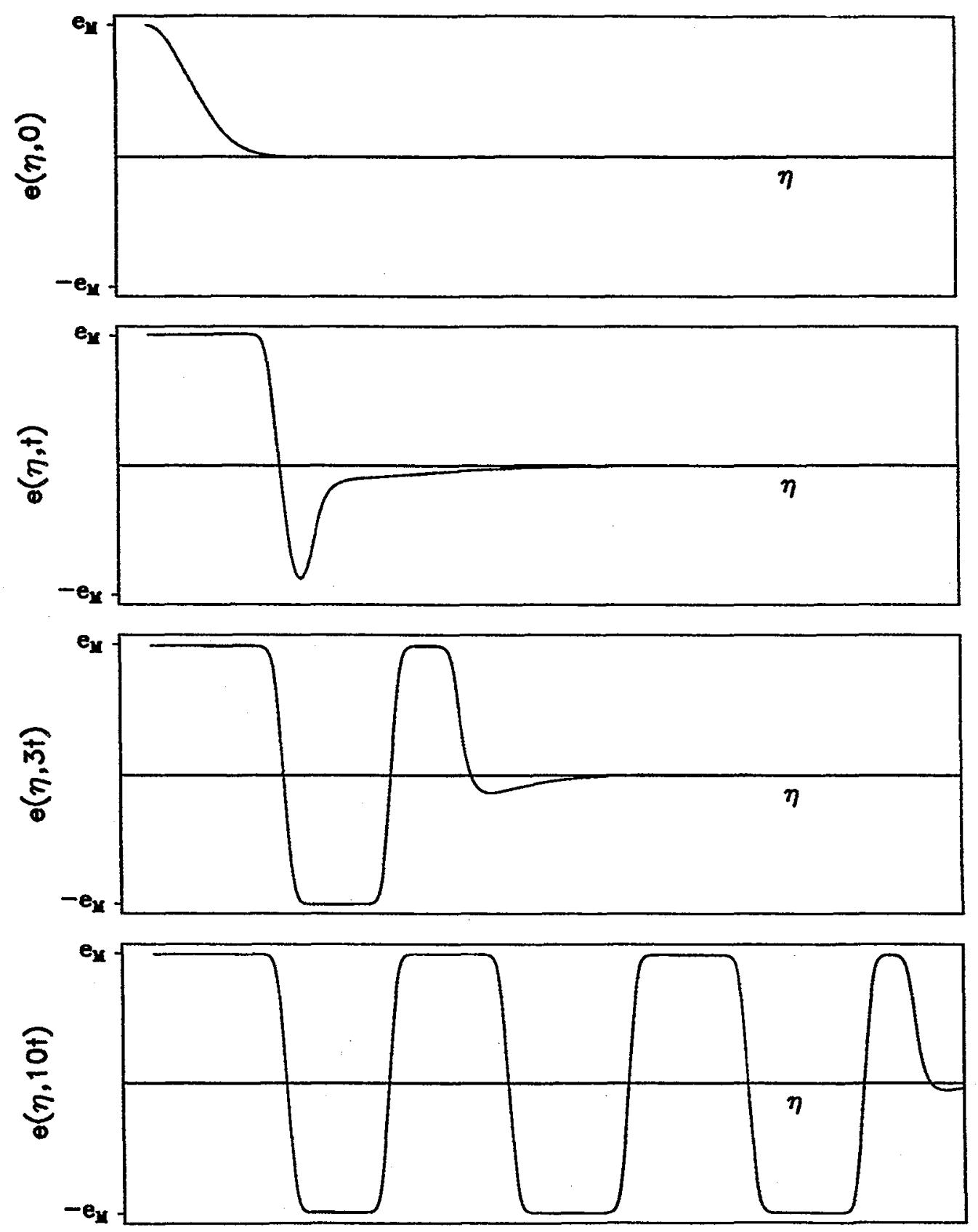

Figure Caption: The above shows the development of a gaussian strain pulse, viz. the martensitic nucleus given in Eq. (14) which is shown in the upper panel, at 3 subsequent times: $t, 3 t$, and $10 t$. The strain $e(\eta, t)$ oscillates between the 2 martensitic strains of the 2 rectangular variants, viz. $\pm e_{M}$. As soon as the nucleus begins to grow, the nucleus begins to twin, viz. just to the right of the nucleus the variant with the opposite strain appears. This sequence perpetuates itself until the growth reaches the boundary. The lower panel clearly represents a twinned, martensitic product phase. 